Sergio RIESE*

\title{
Descrizione di una nuova specie di Pectocera Hope del sud della Cina: Pectocera hainana n. sp. (Coleoptera, Elateridae, Pityobiinae)
}

\begin{abstract}
Riassunto: Viene descritta una nuova specie di Elateridae del sud della Cina (Isola di Hainan): Pectocera hainana n. sp. (ð̋ㅇ) (Coleoptera: Elateridae, Pityobiinae), loc. typ.: Cina - Hainan, Sanya city, mt. Jiangfengling. Presenta lo stesso habitus di P. jiangxiana Kishii \& Jiang, 1994, ma è facilmente distinguibile per la colorazione, gli angoli posteriori del pronoto carenati e lo scutello submitrale nettamente inciso alla base.
\end{abstract}

\begin{abstract}
Description of a new species of Elateridae from southern China (Hainan island): Pectocera hainana $n$. sp. (Coleoptera: Elateridae, Pityobiinae).

A new species of click beetle from southern Cina (Hainan island) is described: Pectocera hainana n. sp. ( $\overbrace{}^{\lambda}$ ), loc. typ.: Cina, Hainan Sanya city, mt. Jiangfengling. It presents the same habitus of $P$. jiangxiana Kishii et Jiang, 1994 from wich is easily distinguishable by body colour, pronotal hind angles carinate and scutellum submitrale shaped, basally incised.
\end{abstract}

Key words: Coleoptera, Elateridae, Pityobiinare, Pectocera, new species, Palearctic region.

\section{INTRODUZIONE}

Il genere Pectocera Hope 1842, è diffuso dal sud-ovest dell'India (Kerala), all'Assam, al Nepal, al sud della Cina, alla Malaysia, a Taiwan, al Giappone e alla Corea. Candèze (1857) ha designato $P$. cantori Hope, 1842 quale specie tipo del genere, assegnandogli originariamente due sole specie: $P$. cantori Hope 1842 e $P$. mellyi Hope 1842, la seconda delle quali successivamente messa in sinonimia con la prima. Attualmente il genere comprende 38 specie.

Avendo ricevuto in studio da Li Jingke una numerosa serie di una Pectocera proveniente dell'isola di Hainan (sud della Cina), ho ritenuto opportuno chiedere un parere al collega Rainer Schimmel, il quale nel 2006 ha descritto 6 specie di Pectocera (P. annapurnica, $P$. godavariana, $P$. kucerai, $P$. mechiana, $P$. mizhilensis e P. myanmarensis), ricevendo da lui conferma che si tratta di una specie non ancora descritta.

\section{Materiali E METODI}

Il materiale esaminato è depositato presso il Museo civico di Storia naturale "G. Doria" di Genova (MSNG) e presso la collezione dell’autore (SRGI).

MisuRE: la lunghezza è misurata lungo la linea mediana, dal margine anteriore della fronte all'apice dell'elitra; la larghezza è misurata nella parte più larga del corpo. La lunghezza del pronoto è misurata sulla linea mediana, mentre la larghezza alla base, prima degli angoli posteriori.

Pectocera hainana n. sp. (Figg.1, 2)

SERIE TIPICA. Holotypus đ̋ Cina, Hainan, Sanya city, mt. Jiangfengling (MSNG).

PARATYPI: 20 지 32 우, idem (MSNG, SRGI).

Dimensioni: lunghezza 24-29 mm ふึग, 27-34 mm

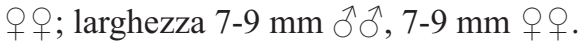

Corpo, zampe e antenne neri, pubescenza dorata pallida, breve, rada, formante delle macchie rotonde sulle elitre. Capo a lati subparalleli, con depressione centrale triangolare, più profonda nei $\widehat{\partial} \widehat{\partial}$, apice arrotondato; punteggiatura forte, profonda, ravvicinata, irregolare, oblunga nei $\widehat{\partial} \widehat{\partial}$, arrotondata nelle $ᄋ$ 우. An-

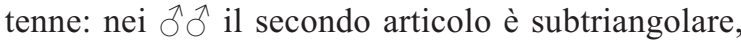
dentato; fortemente lamellate a partire dal terzo articolo, superano gli angoli posteriori del pronoto di circa tre antennomeri e si estendono poco oltre la metà delle elitre. Nelle $q q$ il secondo articolo è subconico, sono dentate a partire dal terzo articolo e superano gli angoli posteriori del pronoto di tre antennomeri circa. Pronoto subtrapezoidale nei $\widehat{\partial} \widehat{o}$, a lati subparalleli nelle

*Sergio Riese, corso Sardegna 46-11d, 16142 Genova, Italia. E-mail: sergio.riese@libero.it 
우우, ristretto all'apice; angoli posteriori divergenti, leggermente carenati, con carena ben distinta dal bordo. Disco munito di un forte e profondo solco longitudinale, che va dalla base all'apice; sono presenti un paio di depressioni tondeggianti vicino all'apice e due depressioni alla base; punteggiatura irregolare per forma e densità, con punti larghi e profondi mescolati a punti molto piccoli, vicini fra loro sul disco, più ravvicinati ai lati e all'apice, piccoli e scarsi alla base. Bordo laterale dentellato. Prosterno e propleure con punteggiatura simile a quella del pronoto, più profonda sul prosterno. Profilo dell'apofisi prosternale formante un angolo ottuso con quello del prosterno. Scutello tozzo, submitrale, nettamente inciso alla base, in rilievo alla base e all'apice.

Elitre convesse, nei $\widehat{\partial} \widehat{\jmath}$ parallele, ristrette a partire circa dai due terzi della lunghezza, nelle 우우 allargate a partire dalla base per circa un quarto della lunghezza, subparallele fino ai due terzi, poi ristrette; presso la sutura punteggiato-striate, munite di una spina terminale laterale; strie leggermente incise alla base, che diventano più profonde procedendo verso l'apice, con punti forti e profondi, distanti fra loro più del loro diametro, interstrie convesse, con punteggiatura irregolare, distanziata e profonda.

Le $ㅇ ㅜ$ si differenziano dai $\widehat{\partial} \hat{o}$ per la forma del pronoto, per le antenne dentate e più brevi e per la forma delle elitre.

Edeago dell'Holotypus come in Fig. 2.

Note COMPARATIVE. $P$. hainana $\mathrm{n}$.sp. si distingue da $P$. jiangxiana Kishii \& Jiang, 1994 per la colorazione, nera anziché marrone, per gli angoli posteriori del pronoto carenati e per la forma dello scutello.

DeRIVATIO NOMINIS. Dal nome dell'Iasola di Hainan, dove è sita la località tipica.

\section{RINGRAZIAMENTI}

Si ringraziano gli amici Rainer Schimmel per gli utili suggeriomenti e Ivo Gudenzi, autore dei disegni.
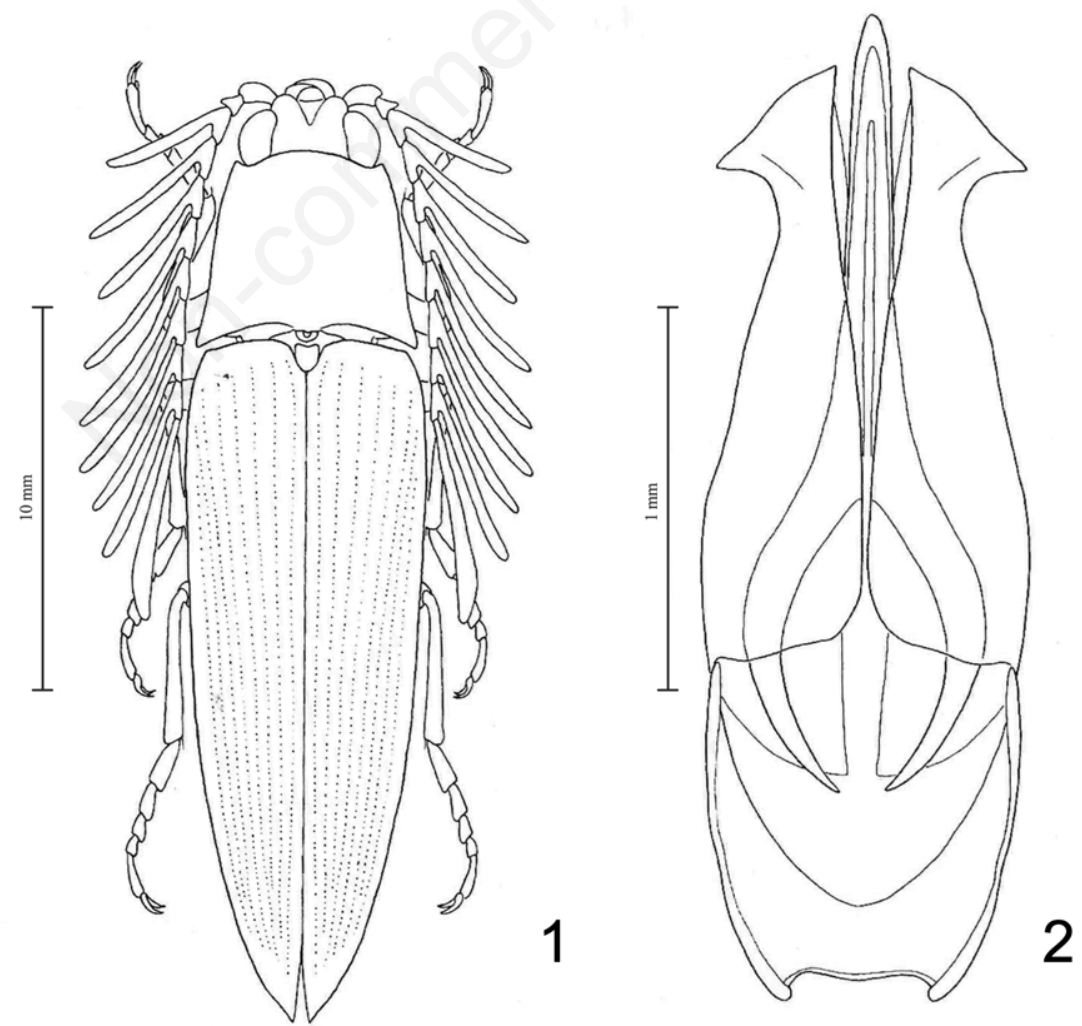

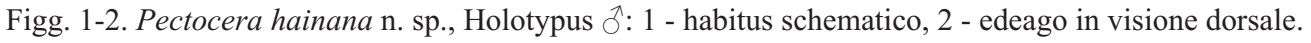




\section{BIBLIOGRAFIA}

CANDÈZE E., 1857 - Monographie des Elatéridés - 1. Mémoires de la Société royale des Sciences de Liège, 12, VIII + 400 pp. KIsHII T., JiANG S.H., 1994 - Notes on the Chinese Elateridae, I (Coleoptera). Entomological Review of Japan, 49: 87-102.

Schimmel R., 2006 - Neue Elateriden-Arten aus der Ampedus- und der Pectocera-gruppe aus Nepal (Insecta: Coleoptera, Elateridae). Veroffentlicuhngen Naturkundemuseum Erfurt, 25: 235-239.

Schimmel R., 2006 - Neue Ampedus-, Denticollis-, Limoniscus-, Pachyderes-, Pectocera- und Pengamethes-Arten aus China, Malaysia, Indonesien und Myanmar (Insecta: Coleoptera, Elateridae). Veroffentlicuhngen Naturkundemuseum Erfurt, 25: 241-259. 